\title{
Convergence of tamed Euler schemes for a class of stochastic evolution equations
}

\author{
István Gyöngy ${ }^{1}$ • Sotirios Sabanis ${ }^{1}$ • David Šiška ${ }^{1}$
}

Received: 8 September 2014 / Published online: 16 November 2015

(C) The Author(s) 2015. This article is published with open access at Springerlink.com

\begin{abstract}
We prove stability and convergence of a full discretization for a class of stochastic evolution equations with super-linearly growing operators appearing in the drift term. This is done by using the recently developed tamed Euler method, which employs a fully explicit time stepping, coupled with a Galerkin scheme for the spatial discretization.
\end{abstract}

Keywords Stochastic evolution equations - Full discretization - Explicit scheme · Convergence $\cdot$ Super-linearly growing operators

Mathematics Subject Classification $60 \mathrm{H} 15 \cdot 65 \mathrm{M} 12$

\section{Introduction}

In this paper we investigate the convergence of full discretizations, explicit in time, of stochastic evolution equations

$$
d u(t)=A u(t) d t+B u(t) d W(t), t \in[0, T]
$$

with the drift term governed by a super-linearly growing operator. When the operator appearing in the drift term grows at most linearly then the classical explicit Euler

David Šiška

d.siska@ed.ac.uk

István Gyöngy

i.gyongy@ed.ac.uk

Sotirios Sabanis

s.sabanis@ed.ac.uk

1 School of Mathematics, University of Edinburgh, Edinburgh, UK 
scheme applied to stochastic evolution equations is convergent (when coupled appropriately with the spatial discretization), see, for example, Gyöngy and Millet [6]. If the operator appearing in the drift term grows faster than linearly then one would, in general, not expect the explicit Euler scheme to be convergent (this is the case even in the setting of fully deterministic evolution equations). Instead, one would typically consider the implicit Euler scheme which is convergent in this situation (see, for example, Gyöngy and Millet [6]). The price one pays is the increased computational effort required at each time step of the numerical scheme.

Hutzenthaler, Jentzen and Kloeden [9] have observed that the absolute moments of explicit Euler approximations for stochastic differential equations with super-linearly growing coefficients may diverge to infinity at finite time. This led to development of "tamed" Euler schemes for stochastic differential equations. This was pioneered in Hutzenthaler, Jentzen and Kloeden [10] and, using different techniques, in Sabanis [16]. A taming-like technique in the form of truncation has been proposed by Roberts and Tweedie [14] in the context of Markov chain Monte Carlo methods. Further work on explicit numerical approximations of stochastic differential equations with super-linearly growing coefficients can be found in Tretyakov and Zhang [19], Hutzenthaler and Jentzen [12], Sabanis [17] as well Dareiotis, Kumar and Sabanis [18].

Moreover Hutzenthaler, Jentzen and Kloeden [11] have demonstrated that to apply multilevel Monte Carlo methods (see Heinrich [7,8] and Giles [5]) to stochastic differential equations with super-linearly growing coefficients one must "tame" the explicit Euler scheme. In this paper we use the idea of "taming" to devise a new type of a convergent explicit scheme for a class of stochastic evolution equations with superlinearly growing operators in the drift term.

The article is organised as follows. In Sect. 2 we introduce the numerical scheme, give the precise assumptions and state the main result in Theorem 2. Essential a priori estimates are proved in Sect. 3. In Sect. 4 we first use the a priori estimates and a compactness argument to extract weakly convergent subsequences and limits of the approximation. The remaining step is to identify the weak limit of the approximation of the nonlinear term with the nonlinear term in the equation. This is done using a monotonicity argument in Sect. 4 where Theorem 2 is finally proved. In Sect. 5 we provide examples of stochastic partial differential equations where the numerical scheme can be applied.

\section{Main results}

Let $T>0$. Let $(\Omega, \mathcal{F}, \mathbb{P})$ be a probability space and let $\left(\mathcal{F}_{t}\right)_{t \in[0, T]}$ be a filtration such that $\mathcal{F}_{0}$ contains all the $\mathbb{P}$-null sets of $\mathcal{F}$.

Let $K>0$ and $p \in[2, \infty)$ be given constants. Let $p^{*}:=p /(p-1)$. For a reflexive, separable Banach space $\left(X,\|\cdot\|_{X}\right)$ let $X^{*}$ and $\|\cdot\|_{X^{*}}$ denote its dual space and the norm on the dual space respectively. For $f \in X^{*}$ and $v \in X$ we use $\langle f, v\rangle$ to denote the duality pairing. By $L^{p}(0, T ; X)$ we denote the Lebesgue-Bochner space of equivalence classes of measurable functions with values in $X$ that satisfy

$$
\|x\|_{L^{p}(0, T ; X)}:=\left(\int_{0}^{T}\|x(t)\|_{X}^{p} d t\right)^{1 / p}<\infty .
$$


By $L^{p}(\Omega ; X)$ we denote the Lebesgue-Bochner space of random variables with values in $X$ and such that the norm

$$
\|x\|_{L^{p}(\Omega ; X)}:=\left(\mathbb{E}\left(\|x\|_{X}^{p}\right)\right)^{1 / p}
$$

is finite. Finally by $\mathcal{L}^{p}(X)$ we denote the Lebesgue-Bochner space of $d t \times \mathbb{P}$ -

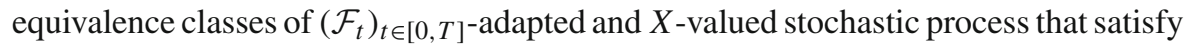

$$
\|x\|_{\mathcal{L}^{p}(X)}:=\left(\mathbb{E} \int_{0}^{T}\|x(t)\|_{X}^{p} d t\right)^{1 / p}<\infty .
$$

We say that an operator $C: X \times \Omega \rightarrow X^{*}$ is measurable with respect to some $\mathcal{G} \subseteq \mathcal{F}$ if for any $v, w \in X$ the real-valued random variable $\langle C v, w\rangle$ is $\mathcal{G}$-measurable.

We assume that, with respect to $\left(\mathcal{F}_{t}\right)_{t \in[0, T]},\left(W_{t}\right)_{t \in[0, T]}$ is a cylindrical $Q$-Wiener process with $Q=I$ on a separable Hilbert space $\left(U,(\cdot, \cdot)_{U},|\cdot|_{U}\right)$. We assume that there are $\left(V_{1},\|\cdot\|_{V_{1}}\right)$ and $\left(V_{2},\|\cdot\|_{V_{2}}\right)$, separable and reflexive Banach spaces that are densely and continuously embedded in $H$, where $(H,(\cdot, \cdot),|\cdot|)$ is a Hilbert space identified with its dual. We thus have two Gelfand triples

$$
V_{i} \hookrightarrow H \hookrightarrow V_{i}^{*}, \quad i \in\{1,2\} .
$$

Let $A_{i}$ with $i \in\{1,2\}$ be operators defined on $V_{i} \times \Omega$ with values in $V_{i}^{*}$. Let $B_{i}$ with $i \in\{1,2\}$ be operators defined on $V_{i} \times \Omega$ with values in $L_{2}(U, H)$, where $L_{2}(U, H)$ is the space of Hilbert-Schmidt operators from $U$ to $H$.

Let $V:=V_{1} \cap V_{2}$ and let the norm in $V$ be given by $\|\cdot\|:=\|\cdot\|_{V_{1}}+\|\cdot\|_{V_{2}}$. Assume that $V$ is separable and dense in both $V_{1}$ and $V_{2}$. Using Gajewski, Gröger and Zacharias [4, Kapitel I,Satz 5.13] one observes that the dual $V^{*}$ of $V$ can be identified with

$$
V_{1}^{*}+V_{2}^{*}:=\left\{f=f_{1}+f_{2}: f_{1} \in V_{1}^{*}, f_{2} \in V_{2}^{*}\right\}
$$

and that for all $f \in V^{*}$

$$
\|f\|_{V *}=\inf \left\{\max \left(\left\|f_{1}\right\|_{V_{1}^{*}},\left\|f_{2}\right\|_{V_{2}^{*}}\right): f=f_{1}+f_{2}, f_{1} \in V_{1}^{*}, f_{2} \in V_{2}^{*}\right\} .
$$

We consider stochastic evolution equations of the form

$$
d u(t)=\left[A_{1} u(t)+A_{2} u(t)\right] d t+\left[B_{1} u(t)+B_{2} u(t)\right] d W(t), t \in[0, T],
$$

where $u(0)=u_{0}$ with $u_{0}$ a given $H$-valued and $\mathcal{F}_{0}$-measurable random variable. Let $A:=A_{1}+A_{2}$ and $B:=B_{1}+B_{2}$. The operator $A$ is defined on $V \times \Omega$ with values in $V^{*}$ and the operator $B$ is defined on $V \times \Omega$ with values in $L_{2}(U, H)$. Then we can write (2.1) as (1.1).

We impose the following assumptions on the operators.

Assumption 1 Let $A_{i}: V_{i} \times \Omega \rightarrow V_{i}^{*}$ be $\mathcal{F}_{0}$-measurable operators for $i \in\{1,2\}$. Let $B_{i}: V_{i} \times \Omega \rightarrow L_{2}(U, H)$ be such that for any $v \in V_{i}, u \in U$ and $h \in H$ the real-valued random variable $\left(\left(B_{i} v\right) u, h\right)$ is $\mathcal{F}_{0}$-measurable for $i \in\{1,2\}$. Moreover assume that the following conditions hold. 


\section{Monotonicity:}

$$
2\langle A v-A w, v-w\rangle+\|B v-B w\|_{L_{2}(U, H)}^{2} \leq K|v-w|^{2} \text { for all } v, w \in V
$$

Coercivity: there is $\mu>0$ such that

$$
2\left\langle A_{1} v, v\right\rangle+\left\|B_{1} v\right\|_{L_{2}(U, H)}^{2} \leq-\mu\|v\|_{V_{1}}^{2}+K\left(1+|v|^{2}\right) \text { for all } v \in V_{1}
$$

and

$$
2\left\langle A_{2} v, v\right\rangle+\left\|B_{2} v\right\|_{L_{2}(U, H)}^{2} \leq K\left(1+|v|^{2}\right) \text { for all } v \in V_{2} .
$$

Growth:

$$
\left\|A_{1} v\right\|_{V_{1}^{*}}^{2} \leq K\left(1+\|v\|_{V_{1}}^{2}\right) \text { for all } v \in V_{1}
$$

and

$$
\left\|A_{2} v\right\|_{V_{2}^{*}}^{p^{*}} \leq K\left(1+\|v\|_{V_{2}}^{p}\right) \text { for all } v \in V_{2}
$$

as well as

$$
\|B v\|_{L_{2}(U, H)}^{2} \leq K\left(1+|v|^{2}\right) \text { for all } v \in H .
$$

Hemicontinuity: for any $v, w$ and $z$ in $V$

$$
\langle A(v+\epsilon w), z\rangle \rightarrow\langle A v, z\rangle \text { as } \epsilon \rightarrow 0 .
$$

We now define what is meant by solution of (1.1).

Definition 1 (Solution) Let $u_{0}$ be an $\mathcal{F}_{0}$-measurable $H$-valued random variable. We

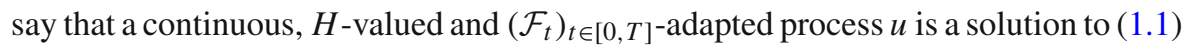
if $u$ is $d t \times \mathbb{P}$ almost everywhere $V$-valued, if $u \in \mathcal{L}^{2}\left(V_{1}\right) \cap \mathcal{L}^{p}\left(V_{2}\right)$ and if for every $t \in[0, T]$ and every $v \in V$, almost surely,

$$
(u(t), v)=\left(u_{0}, v\right)+\int_{0}^{t}\langle A u(s), v\rangle d s+\int_{0}^{t}(v, B u(s) d W(s)) .
$$

To the best knowledge of the authors, existence and uniqueness has not been proved for this class of stochastic evolution equations. Pardoux [13] considers the situation where the stochastic evolution equation is governed by a sum of monotone, coercive and hemicontinuous operators satisfying certain growth condition. However the operator $A_{2}$ in our case only satisfies a type of "degenerate" coercivity condition. Hence the existence theorem from Pardoux [13] does not apply. We prove that a solution to (1.1) must be unique in Theorem 1 and we prove existence of the solution in Theorem 2.

Theorem 1 (Uniqueness) The solution of (1.1), specified by Definition 1, is unique, provided that the Growth and Monotonicity conditions in Assumption 1 are satisfied.

We prove Theorem 1 in Sect. 4. Let us now describe the discretization scheme for the stochastic evolution Eq. (2.1). For the space discretization let $\left(V_{m}\right)_{m \in \mathbb{N}}$ be a Galerkin scheme for $V$. To be precise we assume that $V_{m} \subseteq V$ are finite dimensional 
spaces with the dimension of $V_{m}$ equal to $m$. We further assume that $V_{m} \subseteq V_{m+1}$ for all $m \in \mathbb{N}$ and that

$$
\lim _{m \rightarrow \infty} \inf \left\{\|v-\varphi\|: \varphi \in V_{m}\right\}=0 \quad \forall v \in V .
$$

(this is referred to as the limited completeness of the Galerkin scheme). We will need the following projection operators.

Assumption 2 For any $m \in \mathbb{N}$ let $\Pi_{m}: V^{*} \rightarrow V_{m}$ satisfy the following:

1. For any $v \in V_{m}, \Pi_{m} v=v$.

2. If $f \in V^{*}$ and $v \in V$ then $\left\langle f, \Pi_{m} v\right\rangle=\left\langle v, \Pi_{m} f\right\rangle$.

3. If $g, h \in H$ then $\left(\Pi_{m} g, h\right)=\left(\Pi_{m} h, g\right)$ and $\left|\Pi_{m} h\right| \leq|h|$.

4. There is a constant, depending on $m$ and denoted by $\mathfrak{c}(m)$, such that

$$
\left|\Pi_{m} f\right|^{2} \leq \mathfrak{c}(m)\|f\|_{V^{*}}^{2} \text { for all } f \in V^{*} .
$$

In applications this assumption is easily satisfied. In particular if $\left\{\varphi_{j} \in V: j=\right.$ $1,2, \ldots\}$ is an orthonormal basis in $H$ then taking $V_{m}:=\operatorname{span}\left\{\varphi_{1}, \ldots, \varphi_{m}\right\}$ is a Galerkin scheme for $V$. Taking $\Pi_{m} f:=\sum_{j=1}^{m}\left\langle f, \varphi_{j}\right\rangle \varphi_{j}$ satisfies the first three conditions in Assumption 2. Moreover, the following holds

$$
\left|\Pi_{m} f\right|^{2}=\left|\sum_{j=1}^{m}\left\langle f, \varphi_{j}\right\rangle \varphi_{j}\right|^{2}=\sum_{j=1}^{m}\left\langle f, \varphi_{j}\right\rangle^{2} \leq\|f\|_{V^{*}}^{2} \sum_{j=1}^{m}\left\|\varphi_{j}\right\|_{V}^{2}=\mathfrak{c}(m)\|f\|_{V^{*}}^{2}
$$

where $\mathfrak{c}(m):=\sum_{j=1}^{m}\left\|\varphi_{j}\right\|_{V}^{2}$. Thus the fourth condition in Assumption 2 is also satisfied. Let $\left\{\chi_{i}\right\}_{i \in \mathbb{N}}$ be an orthonormal basis of $U$. Fix $k \in \mathbb{N}$ and define

$$
W_{k}(t):=\sum_{j=1}^{k}\left(W(t), \chi_{j}\right)_{U} \chi_{j}
$$

For the time discretization take $n \in \mathbb{N}$, let $\tau_{n}:=T / n$ and define the grid points on an equidistant grid as $t_{i}^{n}:=\tau_{n} i, i=0,1, \ldots, n$. Further consider some sequence $\left(\left(n_{\ell}, m_{\ell}, k_{\ell}\right)\right)_{\ell \in \mathbb{N}}$ such that $n_{\ell}, m_{\ell}$ and $k_{\ell}$ all go to infinity as $\ell \rightarrow \infty$.

Let $c$ denote a generic positive constant that may depend on $T$, on the constants arising in the continuous embeddings $V_{i} \hookrightarrow H \hookrightarrow V_{i}^{*}, i=1,2$ and on the constants arising in Assumptions 1 and 3 but that is always independent of the discretization parameters $m, k$ and $n$. Define $\kappa_{n_{\ell}}(t)=t_{i}^{n_{\ell}}$ if $t \in\left[t_{i}^{n_{\ell}}, t_{i+1}^{n_{\ell}}\right)$ for $i=0, \ldots, n_{\ell}-1$ and $\kappa_{n_{\ell}}(T)=T$. Fix some $\ell \in \mathbb{N}$ (and hence $m_{\ell}, n_{\ell}$ and $k_{\ell}$ ). Let $u_{\ell}(0)$ be a $V_{m_{\ell}}$ valued $\mathcal{F}_{0}$-measurable approximation of $u_{0}$. For example we can take $u_{\ell}(0):=\Pi_{m_{\ell}} u_{0}$ but other approximations are possible. For $t>0$ we define a process $u_{\ell}$ by

$$
\begin{aligned}
u_{\ell}(t)= & u_{\ell}(0)+\int_{0}^{t} \Pi_{m_{\ell}}\left[A_{1} u_{\ell}\left(\kappa_{n_{\ell}}(s)\right)+A_{2, \ell} u_{\ell}\left(\kappa_{n_{\ell}}(s)\right)\right] d s \\
& +\int_{0}^{t} \Pi_{m_{\ell}} B u_{\ell}\left(\kappa_{n_{\ell}}(s)\right) d W_{k_{\ell}}(s),
\end{aligned}
$$


where we use the "tamed" operator $A_{2, \ell}$ defined by

$$
A_{2, \ell} v:=\frac{1}{1+n_{\ell}-1 / 2\left|\Pi_{m_{\ell}} A_{2} v\right|} A_{2} v
$$

for any $v \in V_{2}$. We will use the following notation: $\bar{u}_{\ell}(t):=u_{\ell}\left(\kappa_{n_{\ell}}(t)\right)$ and $a_{\ell}(v)$ $:=\Pi_{m_{\ell}}\left[A_{1} v+A_{2, \ell} v\right]$. Then (2.2) is equivalent to

$$
u_{\ell}(t)=u_{\ell}(0)+\int_{0}^{t} a_{\ell}\left(\bar{u}_{\ell}(s)\right) d s+\int_{0}^{t} \Pi_{m_{\ell}} B \bar{u}_{\ell}(s) d W_{k_{\ell}}(s) .
$$

In particular at the time-grid points we have, for $i=0,1, \ldots, n_{\ell}-1$,

$$
u_{\ell}\left(t_{i+1}^{n_{\ell}}\right)=u_{\ell}\left(t_{i}^{n_{\ell}}\right)+a_{\ell}\left(u_{\ell}\left(t_{i}^{n_{\ell}}\right)\right) \tau_{n_{\ell}}+\Pi_{m_{\ell}} B u_{\ell}\left(t_{i}^{n_{\ell}}\right) \Delta W_{k_{\ell}}\left(t_{i+1}\right)
$$

where $\Delta W_{k_{\ell}}\left(t_{i+1}^{n_{\ell}}\right):=W_{k_{\ell}}\left(t_{i+1}^{n_{\ell}}\right)-W_{k_{\ell}}\left(t_{i}^{n_{\ell}}\right)$. This in turn is equivalent to

$$
\frac{u_{\ell}\left(t_{i+1}^{n_{\ell}}\right)-u_{\ell}\left(t_{i}^{n_{\ell}}\right)}{\tau_{n_{\ell}}}=a_{\ell}\left(u_{\ell}\left(t_{i}^{n_{\ell}}\right)\right)+\Pi_{m_{\ell}} B u_{\ell}\left(t_{i}^{n_{\ell}}\right) \frac{\Delta W_{k_{\ell}}\left(t_{i+1}^{n_{\ell}}\right)}{\tau_{n_{\ell}}} .
$$

We list below the properies which are satisfied by the tamed operator $A_{2, \ell}$. These are consequences of its structure and the assumed properties of $A_{2}$. For brevity let, for any $v \in V_{2}$,

$$
T_{\ell}(v):=\frac{1}{1+n_{\ell}^{-1 / 2}\left|\Pi_{m_{\ell}} A_{2} v\right|} .
$$

Then for any $v \in V_{2}$,

$$
\left|\Pi_{m_{\ell}} A_{2, \ell} v\right|=T_{\ell}(v)\left|\Pi_{m_{\ell}} A_{2} v\right| \leq n_{\ell}^{1 / 2}
$$

and also, using the Growth assumption on $A_{2}$,

$$
\left\|A_{2, \ell} v\right\|_{V_{2}^{*}}^{p^{*}}=T_{\ell}(v)^{p^{*}}\left\|A_{2} v\right\|_{V_{2}^{*}}^{p^{*}} \leq K\left(1+\|v\|_{V_{2}}^{p}\right) \text {. }
$$

Furthermore, using the Coercivity assumption on $A_{2}$, we note that for all $v \in V_{m_{\ell}}$ we have

$$
2\left\langle A_{2, \ell} v, v\right\rangle=2 T_{\ell}(v)\left\langle A_{2} v, v\right\rangle \leq K\left(1+|v|^{2}\right) .
$$

Thus the weaker coercivity assumption that has been made about $A_{2}$ is retained. Consider, for a moment, that $A_{2}$ satisfies the "usual" coercivity condition

$$
2\left\langle A_{2} v, v\right\rangle+\left\|B_{2} v\right\|_{L_{2}(U, H)}^{2} \leq-\mu\|v\|_{V_{2}}^{p}+K\left(1+|v|^{2}\right) \text { for all } v \in V_{2}
$$

We see that in this case the best coercivity we can get from this for $A_{2, \ell}$ is again only (2.8). Hence to obtain the necessary a priori estimates we will need an interpolation inequality between $V_{2}$ and $V_{1}$ with $H$. 
Assumption 3 There are constants $\lambda \in[0,2 / p)$ and $\Lambda>0$ such that for any $v \in V$

$$
\|v\|_{V_{2}} \leq \Lambda\|v\|_{V_{1}}^{\lambda}|v|^{1-\lambda}
$$

Note that in order to overcome the difficulty with coercivity it would suffice to have Assumption 3 satisfied with $\lambda \in[0,2 / p]$. However monotonicity of $A_{2}$ is not preserved by taming. To overcome this we will need to show that $A_{2, \ell} \bar{u}_{\ell}-A_{2} \bar{u}_{\ell} \rightarrow 0$ in $\mathcal{L}^{p *}\left(V_{2}^{*}\right)$. To achieve this we use the fact that $\lambda \in[0,2 / p)$ in Lemma 4 and the following observation: Assumption 3 implies that there is $\eta>0$ such that

$$
\|v\|_{V_{2}}^{p(1+\eta)} \leq c\|v\|_{V_{1}}^{2}|v|^{\rho}
$$

where $\rho:=(1-\lambda) p(1+\eta)$. From this it follows that if $v \in L^{2}\left(\Omega ; L^{2}\left(0, T ; V_{1}\right)\right)$ and $v \in L^{2 \rho}\left(\Omega ; L^{\infty}(0, T ; H)\right)$ then

$$
\mathbb{E} \int_{0}^{T}\|v(s)\|_{V_{2}}^{p(1+\eta)} d s \leq c\left[\mathbb{E} \sup _{s \leq t}|v(s)|^{2 \rho}+\mathbb{E}\left(\int_{0}^{T}\|v(s)\|_{V_{1}}^{2} d s\right)^{2}\right] .
$$

Thus we see that Assumption 3 allows us to control the approximate solution in the $L^{p(1+\eta)}\left((0, T) \times \Omega ; V_{2}\right)$ norm, provided that we can control the approximate solution in the norms of $L^{2}\left(\Omega ; L^{2}\left(0, T ; V_{1}\right)\right)$ and $L^{2 \rho}\left(\Omega ; L^{\infty}(0, T ; H)\right)$.

Let us take $q_{0}:=\max (4,2 \rho)$. Now we can state the main result of this paper.

Theorem 2 Let Assumptions 1,2 and 3 be satisfied. Let $u_{0} \in L^{q_{0}}(\Omega ; H)$ and let $u_{\ell}(0) \rightarrow u_{0}$ in $L^{q_{0}}(\Omega ; H)$. Assume that $\frac{\mathfrak{c}\left(m_{\ell}\right)}{n_{\ell}} \rightarrow 0$ as $\ell \rightarrow \infty$. Then there exists a unique solution $u$ to $(1.1)$ and $\bar{u}_{\ell} \rightarrow u$ in $\mathcal{L}^{2}\left(V_{1}\right)$ and in $\mathcal{L}^{p}\left(V_{2}\right)$ and $u_{\ell}(T) \rightarrow u(T)$ in $L^{2}(\Omega ; H)$ as $\ell \rightarrow \infty$.

In Sect. 5 we provide examples of stochastic partial differential equations where Theorem 2 can be applied. We also compute $\mathfrak{c}(m)$ in case of the spectral Galerkin method to make the implications of the space-time coupling constraint more explicit. The crucial point is that the requirement is no more onerous than in the case of equations with operators growing at most linearly.

\section{A priori estimates}

We start with an important observation that allows us to use standard results on bounds of stochastic integrals driven by finite dimensional Wiener processes.

Remark 1 Recall that $\left(\chi_{j}\right)_{j \in \mathbb{N}}$ is an orthonormal basis in $U$. Moreover recall that $\bar{u}_{\ell}(t):=u_{\ell}\left(\kappa_{n_{\ell}}(t)\right)$ and that $a_{\ell}(v):=\Pi_{m_{\ell}}\left[A_{1} v+A_{2, \ell} v\right]$. For each $j \in \mathbb{N}$ a Wiener processes $\mathcal{W}_{j}$ is obtained by taking $\mathcal{W}_{j}(t):=\left(W(t), \chi_{j}\right)_{U}$. If $i \neq j$ then $\mathcal{W}_{i}$ and $\mathcal{W}_{j}$ are independent. Furthermore (2.4) is equivalent to

$$
u_{\ell}(t)=u_{\ell}(0)+\int_{0}^{t} a_{\ell}\left(\bar{u}_{\ell}(s)\right) d s+\sum_{j=1}^{k_{\ell}} \int_{0}^{t} \Pi_{m_{\ell}} B \bar{u}_{\ell}(s) \chi_{j} d \mathcal{W}_{j}(s) .
$$


Fix $\ell \in \mathbb{N}$ (and thus $k_{\ell}, m_{\ell}$ and $n_{\ell}$ are also fixed). Then using the Growth assumptions on $A_{1}$ and $B$, Assumption 2 as well as (2.6) one observes that $\left|a_{\ell}(v)\right|^{2} \leq 2 \mathfrak{c}(m) K(1+$ $\left.|v|^{2}\right)+2 n_{\ell}$ and $\left|\Pi_{m_{\ell}} B v \chi_{j}\right|^{2} \leq K\left(1+|v|^{2}\right)$. Hence one knows that, for $q \geq 1$,

$$
\mathbb{E} \sup _{0 \leq t \leq T}\left|u_{\ell}(t)\right|^{q}<\infty
$$

provided that $\mathbb{E}\left|u_{\ell}(0)\right|^{q}<\infty$. Clearly, at this point, one cannot claim that this bound is independent of $\ell$.

One applies Itô's formula to (2.4) to obtain

$$
\begin{aligned}
\left|u_{\ell}(t)\right|^{2}= & \left|u_{\ell}(0)\right|^{2}+\int_{0}^{t}\left[2\left\langle a_{\ell}\left(\bar{u}_{\ell}(s)\right), u_{\ell}(s)\right\rangle+\sum_{j=1}^{k_{\ell}}\left|\Pi_{m_{\ell}} B \bar{u}_{\ell}(s) \chi_{j}\right|^{2}\right] d s \\
& +\int_{0}^{t} 2\left(B \bar{u}_{\ell}(s), u_{\ell}(s) d W_{k_{\ell}}(s)\right)
\end{aligned}
$$

which can be rewritten as

$$
\begin{aligned}
\left|u_{\ell}(t)\right|^{2}= & \left|u_{\ell}(0)\right|^{2}+\int_{0}^{t}\left[2\left\langle a_{\ell}\left(\bar{u}_{\ell}(s)\right), \bar{u}_{\ell}(s)\right\rangle+\sum_{j=1}^{k_{\ell}}\left|\Pi_{m_{\ell}} B \bar{u}_{\ell}(s) \chi_{j}\right|^{2}\right] d s \\
& +2 \int_{0}^{t}\left\langle a_{\ell}\left(\bar{u}_{\ell}(s)\right), u_{\ell}(s)-\bar{u}_{\ell}(s)\right\rangle d s \\
& +\int_{0}^{t} 2\left(B \bar{u}_{\ell}(s), u_{\ell}(s) d W_{k_{\ell}}(s)\right),
\end{aligned}
$$

in order to apply the coercivity assumption so as to obtain the a priori estimates for the discretized equation.

First we concentrate on the term that arises from the "correction" that one has to make to use the coercivity assumption due to the use of an explicit scheme.

Lemma 1 Let the Growth condition in Assumption 1 be satisfied. Let Assumption 2 hold. Let $q \geq 1$ be given. Then

$$
\begin{aligned}
& \mathbb{E}\left(\frac{1}{\tau_{n_{\ell}}} \int_{0}^{t}\left|u_{\ell}(s)-\bar{u}_{\ell}(s)\right|^{2} d s\right)^{q} \\
& \leq c_{T, q}\left(1+\left(\mathfrak{c}(m) \tau_{n_{\ell}}\right)^{q} \mathbb{E}\left(\int_{0}^{t}\left\|\bar{u}_{\ell}(s)\right\|_{V_{1}}^{2} d s\right)^{q}+\mathbb{E} \int_{0}^{t}\left|\bar{u}_{\ell}(s)\right|^{2 q} d s\right)
\end{aligned}
$$

and

$$
\begin{aligned}
& \mathbb{E}\left(\int_{0}^{t}\left|a_{\ell}\left(\bar{u}_{\ell}(s)\right) \| u_{\ell}(s)-\bar{u}_{\ell}(s)\right| d s\right)^{q} \\
& \leq c_{T, q}\left(1+\left(\mathfrak{c}(m) \tau_{n_{\ell}}\right)^{q} \mathbb{E}\left(\int_{0}^{t}\left\|\bar{u}_{\ell}(s)\right\|_{V_{1}}^{2} d s\right)^{q}+\mathbb{E} \int_{0}^{t}\left|\bar{u}_{\ell}(s)\right|^{2 q} d s\right) .
\end{aligned}
$$


Proof From (2.2) it is clear that

$$
\begin{aligned}
I_{1, \ell}(t) & :=\mathbb{E}\left(\frac{1}{\tau_{n_{\ell}}} \int_{0}^{t}\left|u_{\ell}(s)-\bar{u}_{\ell}(s)\right|^{2} d s\right)^{q} \\
& =\mathbb{E}\left(\int_{0}^{t} \frac{1}{\tau_{n_{\ell}}}\left|\int_{\kappa_{n_{\ell}}(s)}^{s} a_{\ell}\left(\bar{u}_{\ell}(r)\right) d r+\int_{\kappa_{n_{\ell}}(s)}^{s} \Pi_{m_{\ell}} B \bar{u}_{\ell}(r) d W_{k_{\ell}}(r)\right|^{2} d s\right)^{q} \\
& \leq 2^{q} \mathbb{E}\left(\frac{1}{\tau_{n_{\ell}}} \int_{0}^{t}\left|\int_{\kappa_{n_{\ell}}(s)}^{s} a_{\ell}\left(\bar{u}_{\ell}(r)\right) d r\right|^{2}+\left|\int_{\kappa_{n_{\ell}}(s)}^{s} \Pi_{m_{\ell}} B \bar{u}_{\ell}(r) d W_{k_{\ell}}(r)\right|^{2} d s\right)^{q} .
\end{aligned}
$$

Applying Hölder's inequality yields

$$
\begin{aligned}
I_{1, \ell}(t) \leq & 2^{q} \mathbb{E}\left(\frac { 1 } { \tau _ { n _ { \ell } } } \int _ { 0 } ^ { t } \left[\left(s-\kappa_{n_{\ell}}(s)\right) \int_{\kappa_{n_{\ell}}(s)}^{s}\left|a_{\ell}\left(\bar{u}_{\ell}(r)\right)\right|^{2} d r\right.\right. \\
& \left.\left.+\left|\int_{\kappa_{n_{\ell}}(s)}^{s} \Pi_{m_{\ell}} B \bar{u}_{\ell}(r) d W_{k_{\ell}}(r)\right|^{2}\right] d s\right)^{q} \\
\leq & c_{q} \mathbb{E}\left(\frac{1}{\tau_{n_{\ell}}} \int_{0}^{t} \tau_{n_{\ell}}^{2}\left|a_{\ell}\left(\bar{u}_{\ell}(s)\right)\right|^{2} d s\right)^{q} \\
& +c_{q} \mathbb{E}\left(\frac{1}{\tau_{n_{\ell}}} \int_{0}^{t}\left|\int_{\kappa_{n_{\ell}}(s)}^{s} \Pi_{m_{\ell}} B \bar{u}_{\ell}(r) d W_{k_{\ell}}(r)\right|^{2} d s\right)^{q} .
\end{aligned}
$$

Using Assumption 2 and (2.6) one obtains

$$
\begin{aligned}
I_{1, \ell}(t) \leq & c_{q} \mathbb{E}\left(\int_{0}^{t} \tau_{n_{\ell}}\left[2 \mathfrak{c}(m)\left\|A_{1} \bar{u}_{\ell}(s)\right\|_{V_{1}^{*}}^{2}+2 n_{\ell}\right] d s\right)^{q} \\
& +c_{q} \mathbb{E}\left(\int_{0}^{t} \frac{1}{\tau_{n_{\ell}}}\left|\int_{\kappa_{n_{\ell}}(s)}^{s} \Pi_{m_{\ell}} B \bar{u}_{\ell}(r) d W_{k_{\ell}}(r)\right|^{2} d s\right)^{q}:=I_{2, \ell}(t)+I_{3, \ell}(t) .
\end{aligned}
$$

The Growth assumption on $A_{1}$ implies that

$$
I_{2, \ell}(t) \leq c_{T, q}\left(1+\left(\mathfrak{c}(m) \tau_{n_{\ell}}\right)^{q} \mathbb{E}\left(\int_{0}^{t}\left\|\bar{u}_{\ell}(s)\right\|_{V_{1}}^{2} d s\right)^{q}\right) .
$$

Using Hölder's inequality leads to

$$
I_{3, \ell}(t) \leq c_{T, q} \mathbb{E} \int_{0}^{t} \frac{1}{\tau_{n_{\ell}}^{q}}\left|\int_{\kappa_{n_{\ell}}(s)}^{s} \Pi_{m_{\ell}} B \bar{u}_{\ell}(r) d W_{k_{\ell}}(r)\right|^{2 q} d s .
$$

Due to Remark 1 and the Growth assumption on $B$ one observes that

$$
I_{3, \ell}(t) \leq c_{T, q} \int_{0}^{t} \frac{1}{\tau_{n_{\ell}}^{q}} \mathbb{E}\left|\int_{\kappa_{n_{\ell}}(s)}^{s}\left\|B \bar{u}_{\ell}(r)\right\|_{L_{2}(U, H)}^{2} d r\right|^{q} d s
$$




$$
\begin{aligned}
& \leq c_{T, q} \mathbb{E} \int_{0}^{t} \frac{1}{\tau_{n_{\ell}}^{q}}\left(s-\kappa_{n_{\ell}}(s)\right)^{q}\left\|B \bar{u}_{\ell}(s)\right\|_{L_{2}(U, H)}^{2 q} d s \\
& \leq c_{T, q}\left(1+\mathbb{E} \int_{0}^{t}\left|\bar{u}_{\ell}(s)\right|^{2 q} d s\right) .
\end{aligned}
$$

This implies (3.2). Moreover Assumption 2 and (2.6) imply that

$$
\begin{aligned}
I_{\ell}(t):= & \mathbb{E}\left(\int_{0}^{t}\left|a_{\ell}\left(\bar{u}_{\ell}(s)\right)\right|\left|u_{\ell}(s)-\bar{u}_{\ell}(s)\right| d s\right)^{q} \\
\leq & 2^{q} \mathbb{E}\left(\int_{0}^{t} \tau_{n_{\ell}}\left|a_{\ell}\left(\bar{u}_{\ell}(s)\right)\right|^{2}+\frac{1}{\tau_{n_{\ell}}}\left|u_{\ell}(s)-\bar{u}_{\ell}(s)\right|^{2} d s\right)^{q} \\
\leq & c_{q} \mathbb{E}\left(\int_{0}^{t}\left[\mathfrak{c}(m) \tau_{n_{\ell}}\left\|A_{1} \bar{u}_{\ell}(s)\right\|_{V_{1}^{*}}^{2}+\tau_{n_{\ell}} n_{\ell}\right] d s\right)^{q} \\
& +c_{q} \mathbb{E}\left(\frac{1}{\tau_{n_{\ell}}} \int_{0}^{t}\left|u_{\ell}(s)-\bar{u}_{\ell}(s)\right|^{2} d s\right)^{q} .
\end{aligned}
$$

Applying the Growth assumption on $A_{1}$ yields

$$
\begin{aligned}
I_{\ell}(t) \leq & c_{T, q}\left(1+\left(\mathfrak{c}(m) \tau_{n_{\ell}}\right)^{q} \mathbb{E}\left(\int_{0}^{t}\left\|\bar{u}_{\ell}(s)\right\|_{V_{1}}^{2} d s\right)^{q}\right) \\
& +c_{q} \mathbb{E}\left(\frac{1}{\tau_{n_{\ell}}} \int_{0}^{t}\left|u_{\ell}(s)-\bar{u}_{\ell}(s)\right|^{2} d s\right)^{q} .
\end{aligned}
$$

Using (3.2) in (3.5) concludes the proof.

Theorem 3 (A priori estimate) Let the Coercivity and Growth conditions in Assumption 1 hold. Let Assumption 2 be satisfied. Let $q \geq 1$ be given and assume that $\mathbb{E}\left|u_{\ell}(0)\right|^{2 q}<c$ and that $u_{\ell}(0)$ is $\mathcal{F}_{0}$-measurable. There is $\epsilon \in(0, \infty)$ such that for all $\ell \in\left\{\ell^{\prime} \in \mathbb{N}: \mathfrak{c}\left(m_{\ell^{\prime}}\right) \tau_{n_{\ell^{\prime}}}<\epsilon\right\}$ we have,

for any $t \in[0, T]$,

$$
\mathbb{E} \sup _{s \in[0, t]}\left|u_{\ell}(s)\right|^{2 q}+\mu^{q} \mathbb{E}\left(\int_{0}^{t}\left\|\bar{u}_{\ell}(s)\right\|_{V_{1}}^{2} d s\right)^{q} \leq c\left(1+\mathbb{E}\left|u_{\ell}(0)\right|^{2 q}\right) .
$$

Proof Applying the Coercivity assumption in (3.1), raising to power $q \geq 1$, taking the supremum over $s \leq t$ and taking the expectation yields

$$
\begin{aligned}
& \mathbb{E} \sup _{s \leq t}\left|u_{\ell}(s)\right|^{2 q}+\mu^{q} \mathbb{E}\left(\int_{0}^{t}\left\|\bar{u}_{\ell}(s)\right\|_{V_{1}}^{2} d s\right)^{q} \leq c_{T, q}\left[1+\mathbb{E}\left|u_{\ell}(0)\right|^{2 q}\right. \\
& +\mathbb{E}\left(\int_{0}^{t}\left|\bar{u}_{\ell}(s)\right|^{2} d s\right)^{q}+\mathbb{E}\left(\int_{0}^{t}\left|a_{\ell}\left(\bar{u}_{\ell}(s)\right)\right|\left|u_{\ell}(s)-\bar{u}_{\ell}(s)\right| d s\right)^{q} \\
& \left.+\mathbb{E} \sup _{s \leq t}\left|\int_{0}^{s}\left(u_{\ell}(s), B \bar{u}_{\ell}(s) d W_{k_{\ell}}(s)\right)\right|^{q}\right] .
\end{aligned}
$$


Using Lemma 1 in (3.6) results in

$$
\begin{aligned}
& \mathbb{E} \sup _{s \leq t}\left|u_{\ell}(s)\right|^{2 q}+\frac{\mu^{q}}{2} \mathbb{E}\left(\int_{0}^{t}\left\|\bar{u}_{\ell}(s)\right\|_{V_{1}}^{2} d s\right)^{q} \\
& \leq c_{T, q}\left[1+\mathbb{E}\left|u_{\ell}(0)\right|^{2 q}+\mathbb{E}\left(\int_{0}^{t}\left|\bar{u}_{\ell}(s)\right|^{2} d s\right)^{q}\right. \\
& \left.\quad+\mathbb{E} \int_{0}^{t}\left|\bar{u}_{\ell}(s)\right|^{2 q} d s+\mathbb{E} \sup _{s \leq t}\left(\int_{0}^{s}\left(u_{\ell}(r), B \bar{u}_{\ell}(r) d W_{k_{\ell}}(r)\right)\right)^{q}\right] .
\end{aligned}
$$

Using Burkholder-Davis-Gundy inequality one obtains

$$
\begin{aligned}
I_{\ell} & :=c_{T, q} \mathbb{E} \sup _{s \leq t}\left|\int_{0}^{s}\left(u_{\ell}(r), B \bar{u}_{\ell}(r) d W_{k_{\ell}}(r)\right)\right|^{q} \\
& \leq\left.\left. c_{T, q} \mathbb{E}\left|\int_{0}^{t}\right| u_{\ell}(s)\right|^{2}\left\|B \bar{u}_{\ell}(s)\right\|_{L_{2}(U, H)}^{2} d s\right|^{q / 2} \\
& \leq c_{T, q} \mathbb{E}\left[\sup _{s \leq t}\left|u_{\ell}(s)\right|^{q}\left(\int_{0}^{t}\left\|B \bar{u}_{\ell}(s)\right\|_{L_{2}(U, H)}^{2} d s\right)^{q / 2}\right] .
\end{aligned}
$$

Young's inequality and the Growth assumption on $B$ imply that

$$
\begin{aligned}
I_{\ell} & \leq \frac{1}{2} \mathbb{E} \sup _{s \leq t}\left|u_{\ell}(s)\right|^{2 q}+c \mathbb{E}\left(\int_{0}^{t}\left\|B \bar{u}_{\ell}(s)\right\|_{L_{2}(U, H)}^{2} d s\right)^{q} \\
& \leq \frac{1}{2} \mathbb{E} \sup _{s \leq t}\left|u_{\ell}(s)\right|^{2 q}+c\left(1+\int_{0}^{t} \mathbb{E} \sup _{r \leq s}\left|u_{\ell}(r)\right|^{2 q} d s\right) .
\end{aligned}
$$

Applying this in (3.7) leads to

$$
\begin{aligned}
& \frac{1}{2} \mathbb{E} \sup _{s \leq t}\left|u_{\ell}(s)\right|^{2 q}+\frac{\mu^{q}}{2} \mathbb{E}\left(\int_{0}^{t}\left\|\bar{u}_{\ell}(s)\right\|_{V_{1}}^{2} d s\right)^{q} \leq c\left[1+\mathbb{E}\left|u_{\ell}(0)\right|^{2 q}\right. \\
& \left.\quad+\int_{0}^{t} \mathbb{E} \sup _{r \leq s}\left|u_{\ell}(r)\right|^{2 q} d s\right] .
\end{aligned}
$$

Application of Gronwall's lemma yields

$$
\mathbb{E} \sup _{s \in[0, t]}\left|u_{\ell}(s)\right|^{2 q}+\mu^{q} \mathbb{E}\left(\int_{0}^{t}\left\|\bar{u}_{\ell}(s)\right\|_{V_{1}}^{2} d s\right)^{q} \leq c\left(1+\mathbb{E}\left|u_{\ell}(0)\right|^{2 q}\right) .
$$

Now we use Theorem 3 and Assumption 3 to obtain the remaining required estimates.

Corollary 1 (Remaining a priori estimates) Let the Growth and Coercivity conditions in Assumption 1 be satisfied. Let Assumptions 2 and 3 hold. Let $u_{\ell}(0)$ be bounded 
in $L^{q_{0}}(\Omega ; H)$, uniformly with respect to $\ell$. There is $\epsilon \in(0, \infty)$ such that for all $\ell \in\left\{\ell^{\prime} \in \mathbb{N}: \mathfrak{c}\left(m_{\ell}^{\prime}\right) \tau_{n_{\ell^{\prime}}}<\epsilon\right\}$ we have

$$
\mathbb{E} \int_{0}^{T}\left\|A_{1} \bar{u}_{\ell}\right\|_{V_{1}^{*}}^{2} d s \leq c, \quad \mathbb{E} \int_{0}^{T}\left\|B \bar{u}_{\ell}\right\|_{L_{2}(U, H)}^{2} d s \leq c .
$$

Furthermore

$$
\mathbb{E} \int_{0}^{T}\left|u_{\ell}(s)-\bar{u}_{\ell}(s)\right|^{2} d s \leq c \tau_{n_{\ell}} .
$$

Finally, for some $\eta>0$,

$$
\mathbb{E} \int_{0}^{T}\left\|\bar{u}_{\ell}\right\|_{V_{2}}^{p(1+\eta)} d s \leq c
$$

and

$$
\mathbb{E} \int_{0}^{T}\left\|A_{2} \bar{u}_{\ell}\right\|_{V_{2}^{*}}^{p^{*}(1+\eta)} d s \leq c, \quad \mathbb{E} \int_{0}^{T}\left\|A_{2, \ell} \bar{u}_{\ell}\right\|_{V_{2}^{*}}^{p^{*}(1+\eta)} d s \leq c .
$$

Proof Inequality (3.9) follows directly from the Growth assumptions on $A_{1}$ and $B$ and from Theorem 3 with $q=1$. Using (3.2), together with Theorem 3 with $q=1$, yields (3.10). Since $u_{\ell}(0)$ is assumed to be bounded in $L^{q_{0}}(\Omega ; H)$, uniformly in $\ell$, one can conclude, using Theorem 3 , that

$$
\mathbb{E} \sup _{s \in[0, t]}\left|u_{\ell}(s)\right|^{2 \rho} \leq c \text { and } \mathbb{E}\left(\int_{0}^{t}\left\|\bar{u}_{\ell}(s)\right\|_{V_{1}}^{2} d s\right)^{2} \leq c .
$$

This, together with Assumption 3, yields (3.11). Finally, (3.11), the assumption on the growth of $A_{2}$ and (2.7) lead to (3.12).

\section{Convergence}

Having obtained the required a priori estimates we can use compactness arguments to extract weakly convergent subsequences of the approximation.

Lemma 2 Let the Growth and Coercivity conditions in Assumption 1 hold. Let Assumptions 2 and 3 be satisfied. Let $u_{\ell}(0) \rightarrow u_{0}$ in $L^{q_{0}}(\Omega ; H)$. Let $\frac{\mathfrak{c}\left(m_{\ell}\right)}{n_{\ell}} \rightarrow 0$ as $\ell \rightarrow \infty$. Then there is a subsequence of the sequence $\ell$, which we denote $\ell^{\prime}$, and $u \in \mathcal{L}^{2}\left(V_{1}\right) \cap \mathcal{L}^{p}\left(V_{2}\right)$ such that, as $\ell^{\prime} \rightarrow \infty$,

$$
\bar{u}_{\ell^{\prime}} \rightarrow u \text { in } \mathcal{L}^{2}\left(V_{1}\right) \text { and in } \mathcal{L}^{p}\left(V_{2}\right)
$$

Furthermore there are $a_{1}^{\infty} \in \mathcal{L}^{2}\left(V_{1}^{*}\right), a_{2}^{\infty} \in \mathcal{L}^{p^{*}}\left(V_{2}^{*}\right)$ and $b^{\infty} \in \mathcal{L}^{2}\left(L_{2}(U, H)\right)$ such that, as $\ell^{\prime} \rightarrow \infty$,

$$
A_{1} \bar{u}_{\ell^{\prime}} \rightarrow a_{1}^{\infty} \text { in } \mathcal{L}^{2}\left(V_{1}^{*}\right), A_{2, \ell^{\prime}} \bar{u}_{\ell^{\prime}} \rightarrow a_{2}^{\infty} \text { in } \mathcal{L}^{p^{*}}\left(V_{2}^{*}\right)
$$

and

$$
B \bar{u}_{\ell^{\prime}} \rightarrow b^{\infty} \text { in } \mathcal{L}^{2}\left(L_{2}(U, H)\right) .
$$


Finally, there is $\xi \in L^{q_{0}}(\Omega ; H)$ such that $\bar{u}_{\ell^{\prime}}(T)=u_{\ell^{\prime}}(T) \rightarrow \xi$ in $L^{q_{0}}(\Omega ; H)$.

Proof The sequence $\left(\bar{u}_{\ell}\right)$ is bounded in $\mathcal{L}^{2}\left(V_{1}\right)$ due to Theorem 3 and in $\mathcal{L}^{p}\left(V_{2}\right)$ due to Corollary 1 . The sequences $\left(A_{1} \bar{u}_{\ell}\right),\left(A_{2, \ell} \bar{u}_{\ell}\right)$ and $\left(B \bar{u}_{\ell}\right)$ are bounded in $\mathcal{L}^{2}\left(V_{1}^{*}\right)$, $\mathcal{L}^{p^{*}}\left(V_{2}^{*}\right)$ and in $\mathcal{L}^{2}\left(L_{2}(U, H)\right)$ respectively, due to Corollary 1 . Finally the sequence $\left(u_{\ell}(T)\right)$ is bounded in $L^{q_{0}}(\Omega ; H)$ due to Theorem 3 .

Since it is assumed that $V_{1}, V_{2}$ are reflexive, it follows that $\mathcal{L}^{2}\left(V_{1}\right)$ and $\mathcal{L}^{p}\left(V_{2}\right)$ are reflexive. A bounded sequence in a reflexive Banach space must have a weakly convergent subsequence (see e.g. Brézis [2, Theorem 3.18]). Applying this to the sequences in question concludes the proof of the lemma.

Let $a^{\infty}:=a_{1}^{\infty}+a_{2}^{\infty}$. Then $a^{\infty} \in \mathcal{L}^{p^{*}}\left(V^{*}\right)$. Due to Lemma $2 a_{\ell}\left(\bar{u}_{\ell}\right) \rightarrow a^{\infty}$ in $\mathcal{L}^{p^{*}}\left(V^{*}\right)$ as $\ell^{\prime} \rightarrow \infty$, provided that $\frac{\mathfrak{c}\left(m_{\ell}\right)}{n_{\ell}} \rightarrow 0$. The following lemma provides the equation satisfied by the weak limits of the approximations.

Lemma 3 Let the Growth and Coercivity conditions in Assumption 1 hold. Let Assumptions 2 and 3 be satisfied. Let $u_{\ell}(0) \rightarrow u_{0}$ in $L^{q_{0}}(\Omega ; H)$. Let $\frac{\mathfrak{c}\left(m_{\ell}\right)}{n_{\ell}} \rightarrow 0$ as $\ell \rightarrow \infty$. Then there is an $H$-valued adapted continuous process $\tilde{u}$ on $[0, T]$ such that $u=\tilde{u} d t \times \mathbb{P}$-almost everywhere on $(0, T) \times \Omega$. Furthermore, for almost every $(t, \omega) \in(0, T) \times \Omega$,

$$
\tilde{u}(t)=u_{0}+\int_{0}^{t} a^{\infty}(s) d s+\int_{0}^{t} b^{\infty}(s) d W(s)
$$

and almost surely

$$
\tilde{u}(T)=u_{0}+\int_{0}^{T} a^{\infty}(s) d s+\int_{0}^{T} b^{\infty}(s) d W(s) .
$$

In the rest of this paper we will write $u$ instead of $\tilde{u}$ for notational simplicity.

Proof Fix $M \in \mathbb{N}$. Let $\varphi$ be a $V_{M}$-valued adapted stochastic process such that $|\varphi(t)|<$ $M$ for all $t \in[0, T]$ and $\omega \in \Omega$. For $g \in U$ let $\tilde{\Pi}_{m} g:=\sum_{j=1}^{m}\left(\chi_{j}, g\right)_{U} \chi_{j}$ and note that for any $v \in V$ one has $B v \tilde{\Pi}_{m} \in L_{2}(U, H)$. From (2.2) one observes that

$$
\begin{aligned}
\left(u_{\ell^{\prime}}(t), \varphi(t)\right) & =\left(u_{\ell^{\prime}}(0), \varphi(t)\right)+\left\langle\int_{0}^{t} a_{\ell^{\prime}}\left(\bar{u}_{\ell^{\prime}}(s)\right) d s, \varphi(t)\right\rangle \\
& +\left(\int_{0}^{t} B \bar{u}_{\ell^{\prime}}(s) \tilde{\Pi}_{m_{\ell^{\prime}}} d W(s), \varphi(t)\right)
\end{aligned}
$$

Let $G: \mathcal{L}^{p^{*}}\left(V^{*}\right) \rightarrow \mathcal{L}^{p^{*}}\left(V^{*}\right)$ be given by $(G v)(t):=\int_{0}^{t} v(s) d s$. Moreover, let $I: \mathcal{L}^{2}\left(L_{2}(U, H)\right) \rightarrow \mathcal{L}^{2}(H)$ be given by $(I v)(t):=\int_{0}^{t} v(s) d W(s)$. Integrating (4.3) from 0 to $T$ and taking the expectation yields 


$$
\begin{aligned}
\mathbb{E} \int_{0}^{T}\left(u_{\ell^{\prime}}(t), \varphi(t)\right) d t= & \mathbb{E} \int_{0}^{T}\left(u_{\ell^{\prime}}(0), \varphi(t)\right) d t+\mathbb{E} \int_{0}^{T}\left\langle\left(G a_{\ell^{\prime}}\left(\bar{u}_{\ell^{\prime}}\right)\right)(t), \varphi(t)\right\rangle d t \\
& +\mathbb{E} \int_{0}^{T}\left(\left(H B \bar{u}_{\ell^{\prime}} \tilde{\Pi}_{m_{\ell^{\prime}}}\right)(t), \varphi(t)\right) d t
\end{aligned}
$$

The operator $G$ is linear and bounded and as such it is weakly-weakly continuous. This operator $I$ is clearly linear. Furthermore, due to Itô's isometry,

$$
\begin{aligned}
\|I v\|_{\mathcal{L}^{2}(H)}^{2} & =\mathbb{E} \int_{0}^{T}|(I v)(s)|^{2} d s \\
& =\int_{0}^{T} \mathbb{E}\left|\int_{0}^{t} v(s) d W(s)\right|^{2} d t \\
& =\mathbb{E} \int_{0}^{T} \int_{0}^{t}|v(s)|^{2} d s d t \leq T\|v\|_{\mathcal{L}^{2}\left(L_{2}(U, H)\right)}^{2}
\end{aligned}
$$

Thus the operator $I$ is also bounded. It follows that $I$ is also weakly-weakly continuous. Therefore, taking the limit as $\ell^{\prime} \rightarrow \infty$ and using Lemma 2, one obtains

$$
\begin{aligned}
\mathbb{E} \int_{0}^{T}(u(t), \varphi(t)) d t= & \mathbb{E} \int_{0}^{T}\left(u_{0}, \varphi(t)\right) d t \\
& +\mathbb{E} \int_{0}^{T}\left\langle\left(G a^{\infty}\right)(t), \varphi(t)\right) d t+\mathbb{E} \int_{0}^{T}\left(\left(I b^{\infty}\right)(t), \varphi(t)\right) d t .
\end{aligned}
$$

This holds for any $\varphi$ as specified at the beginning of the proof. By letting $M \rightarrow \infty$ and using the limited completeness of the Galerkin scheme it follows that this also holds for any $\varphi \in \mathcal{L}^{p}(V)$. Thus

$$
u(t)=u_{0}+\int_{0}^{t} a^{\infty}(s) d s+\int_{0}^{t} b^{\infty}(s) d W(s)
$$

holds for almost all $(t, \omega) \in(0, T) \times \Omega$.

Let $\varphi$ be a $V_{M}$-valued and $\mathcal{F}_{T}$-measurable random variable such that $\mathbb{E}\|\varphi\|_{V}^{2}<\infty$. Setting $t=T$ in (4.3) and taking the expectation yields

$$
\begin{aligned}
\mathbb{E}\left(u_{\ell^{\prime}}(T), \varphi\right)= & \mathbb{E}\left(u_{\ell^{\prime}}(0), \varphi\right) \\
& +\mathbb{E}\left\langle\left(G a_{\ell^{\prime}}\left(\bar{u}_{\ell^{\prime}}\right)\right)(T), \varphi\right\rangle+\mathbb{E}\left(\left(I B \bar{u}_{\ell^{\prime}} \tilde{\Pi}_{m^{\prime}}\right)(T), \varphi\right) .
\end{aligned}
$$

Let $\ell^{\prime} \rightarrow \infty$. The weak-weak continuity of the operators $G$ and $I$, together with Lemma 2, implies that

$$
\mathbb{E}(\xi, \varphi)=\mathbb{E}\left(u_{0}, \varphi\right)+\mathbb{E}\left(\left(G a^{\infty}\right)(T), \varphi\right)+\mathbb{E}\left(\left(I b^{\infty}\right)(T), \varphi\right) .
$$

Letting $M \rightarrow \infty$ and again using the limited completeness of the Galerkin scheme shows that the above equality holds for any $\mathcal{F}_{T}$-measurable $\varphi \in L^{2}(\Omega ; V)$. If one 
now applies Itô's formula to (4.4) then one obtains an adapted process $\tilde{u}$ with paths in $C([0, T] ; H)$ that is equal to $u$ almost surely. Furthermore, for any $\varphi \in L^{2}(\Omega ; V)$ and due to continuity of $\tilde{u}$,

$$
\begin{aligned}
\mathbb{E}(\xi-\tilde{u}(T), \varphi) & =\lim _{t \rightarrow T} \mathbb{E}(\xi-\tilde{u}(t), \varphi) \\
& =\lim _{t \rightarrow T} \mathbb{E}\left\langle\int_{t}^{T} a^{\infty}(s) d s+\int_{t}^{T} b^{\infty}(s) d W(s), \varphi\right\rangle=0 .
\end{aligned}
$$

Thus $\xi=\tilde{u}(T)$. This together with (4.5) implies (4.2).

All that remains to be done to prove Theorem 2 is to identify $a^{\infty}$ with $A u$ and $b^{\infty}$ with $B u$ and to show strong convergence of $u_{\ell}(T)$ to $u(T)$. To that end we would like to use monotonicity of $A$. In order to overcome the difficulty arising from the fact that the tamed operator $A_{2, \ell}$ does not preserve the monotonicity property of $A_{2}$ we need the following lemma.

Lemma 4 Let the Growth and Coercivity conditions in Assumption 1 hold. Let Assumptions 2 and 3 be satisfied. Let $u_{\ell}(0) \rightarrow u_{0}$ in $L^{q_{0}}(\Omega ; H)$. Let $\frac{\mathfrak{c}\left(m_{\ell}\right)}{n_{\ell}} \rightarrow 0$ as $\ell \rightarrow \infty$. Then

$$
\mathbb{E} \int_{0}^{T}\left\|A_{2} \bar{u}_{\ell}(s)-A_{2, \ell} \bar{u}_{\ell}(s)\right\|_{V_{2}^{*}}^{p^{*}} d s \rightarrow 0 \text { as } \ell \rightarrow \infty .
$$

Proof Consider some $M>0$. Recall that $T_{\ell}$ is given by (2.5). Then

$$
\begin{aligned}
I_{\ell}:= & \mathbb{E} \int_{0}^{T}\left\|A_{2, \ell} \bar{u}_{\ell}(s)-A_{2} \bar{u}_{\ell}(s)\right\|_{V_{2}^{*}}^{p^{*}} d s \\
= & \mathbb{E} \int_{0}^{T}\left(1-T_{\ell}\left(\bar{u}_{\ell}(s)\right)\right)^{p^{*}}\left\|A_{2} \bar{u}_{\ell}(s)\right\|_{V_{2}^{*}}^{p^{*}} \mathbb{1}_{\left\{\left\|A_{2} \bar{u}_{\ell}(s)\right\|_{\left.V_{2}^{*} \leq M\right\}} d s\right.} \\
& +\mathbb{E} \int_{0}^{T}\left(1-T_{\ell}\left(\bar{u}_{\ell}(s)\right)\right)^{p^{*}}\left\|A_{2} \bar{u}_{\ell}(s)\right\|_{V_{2}^{*}}^{p^{*}} \mathbb{1}_{\left\{\left\|A_{2} \bar{u}_{\ell}(s)\right\|_{\left.V_{2}^{*}>M\right\}} d s\right.} \\
= & : I_{1, \ell, M}+I_{2, \ell, M} .
\end{aligned}
$$

It is observed that

$$
\begin{aligned}
I_{1, \ell, M} & \leq \mathbb{E} \int_{0}^{T} \frac{n_{\ell}^{-1 / 2}\left|\Pi_{m_{\ell}} A_{2} \bar{u}_{\ell}(s)\right|}{1+n_{\ell}^{-1 / 2}\left|\Pi_{m_{\ell}} A_{2} \bar{u}_{\ell}(s)\right|}\left\|A_{2} \bar{u}_{\ell}(s)\right\|_{V_{2}^{*}}^{p^{*}} \mathbb{1}_{\left\{\left\|A_{2} \bar{u}_{\ell}(s)\right\|_{\left.V_{2}^{*} \leq M\right\}} d s\right.} \\
& \leq \mathbb{E} \int_{0}^{T} \frac{\tau_{n_{\ell}}^{1 / 2} T^{-1 / 2} \mathfrak{c}(m)^{1 / 2} M}{1+n_{\ell}^{-1 / 2}\left|\Pi_{m_{\ell}} A_{2} \bar{u}_{\ell}(s)\right|} M^{p^{*}} d s \leq\left(\mathfrak{c}(m) \tau_{n_{\ell}}\right)^{1 / 2} T^{1 / 2} M^{1+p^{*}}
\end{aligned}
$$

Recall that due to Corollary 1 one knows that

$$
\mathbb{E} \int_{0}^{T}\left\|A_{2} \bar{u}_{\ell}(s)\right\|_{V_{2}^{*}}^{p^{*}(1+\eta)} d s<c
$$


with $c$ independent of $\ell$. Thus the sequence $\left(\left\|A_{2} \bar{u}_{\ell}\right\|_{V_{2}^{*}}^{p^{*}}\right)_{\ell \in \mathbb{N}}$ is uniformly integrable on $(0, T) \times \Omega$ with respect to $d t \times P$. Hence for any $\epsilon>0$ there exists $M$ such that $I_{2, \ell, M}<\epsilon / 2$ for all $\ell$. Finally, since $\frac{\mathfrak{c}\left(m_{\ell}\right)}{n_{\ell}} \rightarrow 0$ as $\ell \rightarrow \infty$, one can choose $\ell$ large such that $I_{1, \ell, M}<\epsilon / 2$.

We now prove Theorem 1 . This is needed to later show that the whole sequence of approximations converges rather than just a subsequence.

Proof of Theorem 1 Assume that $u_{1}$ and $u_{2}$ are two distinct solutions to (1.1) such that $u_{1}(0)=u_{2}(0)=u_{0}$. One would now like to apply Itô's formula for the square of the norm from Pardoux [13, Chapitre 2, Theoreme 5.2]. To that end one immediately observes that $u_{1}-u_{2} \in \mathcal{L}^{2}\left(V_{1}\right) \cap \mathcal{L}^{p}\left(V_{2}\right)$ and that $u_{1}(0)-u_{2}(0)=0 \in L^{2}(\Omega ; H)$. Moreover

$$
\left\|A u_{1}-A u_{2}\right\|_{\mathcal{L}^{2}\left(V_{1}^{*}\right)+\mathcal{L} p^{*}\left(V_{2}^{*}\right)}=\left\|A_{1} u_{1}-A_{1} u_{2}\right\|_{\mathcal{L}^{2}\left(V_{1}^{*}\right)}+\left\|A_{2} u_{1}-A_{2} u_{2}\right\|_{\mathcal{L} p^{*}\left(V_{2}^{*}\right)} \text {. }
$$

Using the Growth assumption on $A_{1}$ one observes that

$$
\begin{aligned}
& \mathbb{E} \int_{0}^{T}\left\|A_{1} u_{1}(s)-A_{1} u_{2}(s)\right\|_{V_{1}^{*}}^{2} d s \leq \mathbb{E} \int_{0}^{T} 2\left[\left\|A_{1} u_{1}(s)\right\|_{V_{1}^{*}}^{2}+\left\|A_{1} u_{2}(s)\right\|_{V_{1}^{*}}^{2}\right] d s \\
& \quad \leq K \mathbb{E} \int_{0}^{T} 2\left[\left(1+\left\|u_{1}(s)\right\|_{V_{1}}^{2}\right)+\left(1+\left\|u_{2}(s)\right\|_{V_{1}}^{2}\right)\right] d s \\
& \quad \leq c\left(1+\left\|u_{1}\right\|_{\mathcal{L}^{2}\left(V_{1}\right)}^{2}+\left\|u_{2}\right\|_{\mathcal{L}^{2}\left(V_{1}\right)}^{2}\right)<\infty .
\end{aligned}
$$

Also, using the Growth assumption on $A_{2}$ one obtains

$$
\mathbb{E} \int_{0}^{T}\left\|A_{2} u_{1}(s)-A_{2} u_{2}(s)\right\|_{V_{2}^{*}}^{p^{*}} d s \leq c\left(1+\left\|u_{1}\right\|_{\mathcal{L}^{p}\left(V_{2}\right)}^{p}+\left\|u_{2}\right\|_{\mathcal{L}^{p}\left(V_{2}\right)}^{p}\right)<\infty
$$

Thus $A u_{1}-A u_{2} \in \mathcal{L}^{2}\left(V_{1}^{*}\right) \cup \mathcal{L}^{p^{*}}\left(V_{2}^{*}\right)$. Finally, using the Growth assumption on $B$ one deduces that $B u_{1}-B u_{2} \in \mathcal{L}^{2}\left(L_{2}(U, H)\right)$. Hence the afromentioned Itô's formula for the square of the norm can be applied, yielding

$$
\begin{aligned}
\left|u_{1}(t)-u_{2}(t)\right|^{2}= & -K \int_{0}^{t} e^{-K s}\left|u_{1}(s)-u_{2}(s)\right|^{2} d s \\
& +\int_{0}^{t} e^{-K s}\left[2\left\langle A u_{1}(s)-A u_{2}(s), u_{1}(s)-u_{2}(s)\right\rangle\right. \\
& \left.+\left\|B u_{1}(s)-B u_{2}(s)\right\|_{L_{2}(U, H)}^{2}\right] d s+M(t),
\end{aligned}
$$

where

$$
M(t):=\int_{0}^{t} e^{-K s}\left(u_{1}(s)-u_{2}(s),\left(B u_{1}(s)-B u_{2}(s)\right) d W(s)\right) .
$$

One then observes, due to the monotonicity of $A: V \times \Omega \rightarrow V^{*}$, that

$$
\left|u_{1}(t)-u_{2}(t)\right|^{2} \leq M(t)
$$


and hence $M$ is non-negative. It is also a real-valued continuous local martingale, and thus a supermartingale. Furthermore it starts from 0 and thus, almost surely, $M(t)=0$ for all $t \in[0, T]$. One thus concludes that $u_{1}(t)=u_{2}(t)$ for all $t \in[0, T]$ almost surely.

Finally we can prove Theorem 2 .

Proof of Theorem 2 Recall that $a_{\ell}(v):=\Pi_{m_{\ell}}\left[A_{1} v+A_{2, \ell} v\right]$. Applying Itô's formula to the scheme (2.2) and taking expectations yields

$$
\begin{aligned}
e^{-K T} \mathbb{E}\left|u_{\ell^{\prime}}(T)\right|^{2}= & \mathbb{E}\left|u_{\ell^{\prime}}(0)\right|^{2}-K \mathbb{E} \int_{0}^{T} e^{-K s}\left|u_{\ell^{\prime}}(s)\right|^{2} d s \\
& +\mathbb{E} \int_{0}^{T} e^{-K s}\left(2\left\langle a_{\ell^{\prime}}\left(\bar{u}_{\ell^{\prime}}(s)\right), \bar{u}_{\ell^{\prime}}(s)\right\rangle+\left\|\Pi_{m_{\ell^{\prime}}} B \bar{u}_{\ell^{\prime}}(s)\right\|_{L_{2}(U, H)}^{2}\right) d s \\
& +\mathbb{E} \int_{0}^{T} 2 e^{-K s}\left\langle a_{\ell^{\prime}}\left(\bar{u}_{\ell^{\prime}}(s)\right), u_{\ell^{\prime}}(s)-\bar{u}_{\ell^{\prime}}(s)\right\rangle d s .
\end{aligned}
$$

Let

$$
I_{1, \ell^{\prime}}:=\mathbb{E} \int_{0}^{T}\left\langle a_{\ell^{\prime}}\left(\bar{u}_{\ell^{\prime}}(s)\right), u_{\ell^{\prime}}(s)-\bar{u}_{\ell^{\prime}}(s)\right\rangle d s .
$$

Using Hölder's inequality results in

$$
I_{1, \ell^{\prime}} \leq\left(\mathbb{E} \int_{0}^{T}\left|a_{\ell}\left(\bar{u}_{\ell^{\prime}}(s)\right)\right|^{2} d s\right)^{1 / 2}\left(\mathbb{E} \int_{0}^{T}\left|u_{\ell^{\prime}}(s)-\bar{u}_{\ell^{\prime}}(s)\right|^{2} d s\right)^{1 / 2}
$$

Using Assumption 2 and Corollary 1 yields

$$
I_{1, \ell^{\prime}} \leq c\left(\mathfrak{c}\left(m_{\ell^{\prime}}\right) \tau_{n_{\ell^{\prime}}}\right)^{1 / 2}
$$

Thus,

$$
\begin{aligned}
e^{-K s} \mathbb{E}\left|u_{\ell^{\prime}}(T)\right|^{2} \leq & \mathbb{E}\left|u_{\ell^{\prime}}(0)\right|^{2}-K \mathbb{E} \int_{0}^{T} e^{-K s}\left|u_{\ell^{\prime}}(s)\right|^{2} d s \\
& +\mathbb{E} \int_{0}^{T} e^{-K s}\left(2\left\langle a_{\ell^{\prime}}\left(\bar{u}_{\ell^{\prime}}(s)\right), \bar{u}_{\ell^{\prime}}(s)\right\rangle+\left\|B \bar{u}_{\ell^{\prime}}(s)\right\|_{L_{2}(U, H)}^{2}\right) d s \\
& +c\left(\mathfrak{c}\left(m_{\ell^{\prime}}\right) \tau_{n_{\ell^{\prime}}}\right)^{1 / 2}
\end{aligned}
$$

and one may proceed with a monotonicity argument. Let $w \in \mathcal{L}^{p}(V)$. Then

$$
\begin{aligned}
& e^{-K T} \mathbb{E}\left|u_{\ell^{\prime}}(T)\right|^{2} \leq \mathbb{E}\left|u_{\ell^{\prime}}(0)\right|^{2}-K \mathbb{E} \int_{0}^{T} e^{-K s}\left|u_{\ell^{\prime}}(s)\right|^{2} d s \\
& \quad+\mathbb{E} \int_{0}^{T} e^{-K s}\left[2\left\langle a_{\ell^{\prime}}\left(\bar{u}_{\ell^{\prime}}(s)\right)-a_{\ell^{\prime}}(w(s)), \bar{u}_{\ell^{\prime}}(s)-w(s)\right\rangle\right. \\
& \left.+2\left\langle a_{\ell^{\prime}}(w(s)), \bar{u}_{\ell^{\prime}}(s)\right)-w(s)\right\rangle+2\left\langle a_{\ell^{\prime}}\left(\bar{u}_{\ell^{\prime}}(s)\right), w(s)\right\rangle
\end{aligned}
$$




$$
\begin{aligned}
& +2\left(B w(s), B \bar{u}_{\ell^{\prime}}(s)\right)_{L_{2}(U, H) \times L_{2}(U, H)}-\|B w(s)\|_{L_{2}(U, H)}^{2} \\
& \left.\left.+\| B \bar{u}_{\ell^{\prime}}(s)\right)-B w(s) \|_{L_{2}(U, H)}^{2}\right] d s+c\left(\mathfrak{c}\left(m_{\ell^{\prime}}\right) \tau_{n_{\ell^{\prime}}}\right)^{1 / 2} .
\end{aligned}
$$

Using the Monotonicity assumption on $A$ one obtains

$$
\begin{aligned}
e^{-K T} \mathbb{E}\left|u_{\ell^{\prime}}(T)\right|^{2} \leq & \mathbb{E}\left|u_{\ell^{\prime}}(0)\right|^{2} \\
& +K \mathbb{E} \int_{0}^{T} e^{-K s}\left(\left|\bar{u}_{\ell^{\prime}}(s)\right|^{2}-\left|u_{\ell^{\prime}}(s)\right|^{2}-2\left(\bar{u}_{\ell^{\prime}}(s), w(s)\right)\right. \\
& \left.+|w(s)|^{2}\right) d s \\
& +\mathbb{E} \int_{0}^{T} e^{-K s}\left\langle A_{2, \ell^{\prime}} \bar{u}_{\ell^{\prime}}(s)-A_{2} \bar{u}_{\ell^{\prime}}(s), \bar{u}_{\ell^{\prime}}(s)-w(s)\right\rangle d s \\
& +\mathbb{E} \int_{0}^{T} e^{-K s}\left\langle A_{2} w(s)-A_{2, \ell^{\prime}} w(s), \bar{u}_{\ell^{\prime}}(s)-w(s)\right\rangle d s \\
& +\mathbb{E} \int_{0}^{T} e^{-K s}\left[2\left\langle a_{\ell^{\prime}}(w(s)), \bar{u}_{\ell^{\prime}}(s)\right)-w(s)\right\rangle \\
& +2\left\langle a_{\ell^{\prime}}\left(\bar{u}_{\ell^{\prime}}(s)\right), w(s)\right\rangle \\
& \left.+2\left(B w(s), B \bar{u}_{\ell}(s)\right)_{L_{2}(U, H) \times L_{2}(U, H)}-\|B w(s)\|_{L_{2}(U, H)}^{2}\right] d s \\
& +c\left(\mathfrak{c}\left(m_{\ell^{\prime}}\right) \tau_{n_{\ell^{\prime}}}\right)^{1 / 2} .
\end{aligned}
$$

Taking limit inferior as $\ell^{\prime} \rightarrow \infty$, using the weak lower-semi-continuity of the norm, Lemmas 2, 4 and Corollary 1, one observes that

$$
\begin{aligned}
e^{-K T} \mathbb{E}|u(T)|^{2} \leq & \mathbb{E}\left|u_{0}\right|^{2}+K \mathbb{E} \int_{0}^{T} e^{-K s}\left[-2(u(s), w(s))+|w(s)|^{2}\right] d s \\
& +\mathbb{E} \int_{0}^{T} e^{-K s}\left[2\langle A w(s), u(s)-w(s)\rangle+2\left\langle a^{\infty}(s), w(s)\right\rangle\right. \\
& \left.+2\left(B w(s), b^{\infty}(s)\right)_{L_{2}(U, H) \times L_{2}(U, H)}-\|B w(s)\|_{L_{2}(U, H)}^{2}\right] d s .
\end{aligned}
$$

Applying Itô's formula to (4.1) and taking expectations yields

$$
\begin{aligned}
e^{-K T} \mathbb{E}|u(T)|^{2}= & \mathbb{E}|u(0)|^{2}-K \mathbb{E} \int_{0}^{T} e^{-K s}|u(s)|^{2} d s \\
& +\mathbb{E} \int_{0}^{T} e^{-K s}\left[2\left\langle a^{\infty}(s), u(s)\right\rangle+\left\|b^{\infty}(s)\right\|_{L_{2}(U, H)}^{2}\right] d s
\end{aligned}
$$

Subtracting this from (4.7) one arrives at

$$
\begin{aligned}
0 \leq & \mathbb{E} \int_{0}^{T} e^{-K s}\left[K|u(s)-w(s)|^{2}+2\langle A w(s), u(s)-w(s)\rangle\right. \\
& \left.+2\left\langle a^{\infty}(s), w(s)-u(s)\right\rangle-\left\|B u(s)-b^{\infty}(s)\right\|_{L_{2}(U, H)}^{2}\right] d s .
\end{aligned}
$$


Note that so far $w$ was arbitrary. It will now be used to identify the nonlinear terms.

First, one takes $w=u$ and observes that,

$$
0 \leq-\mathbb{E} \int_{0}^{T} e^{-K s}\left\|B u(s)-b^{\infty}(s)\right\|_{L_{2}(U, H)}^{2} d s \leq 0
$$

which implies $b^{\infty}=B u$. Next, one sets $w=u+\epsilon z$ with $\epsilon>0$ and $z \in \mathcal{L}^{p}(V)$ in (4.9). Dividing by $\epsilon>0$ leads to

$$
0 \leq \mathbb{E} \int_{0}^{T} e^{-K s}\left[K \epsilon|z|^{2}+2\langle A(u(s)+\epsilon z(s)),-z(s)\rangle+2\left\langle a^{\infty}(s), z(s)\right\rangle\right] d s .
$$

Using hemicontinuity of $A$ while letting $\epsilon \rightarrow 0$ results in

$$
\mathbb{E} \int_{0}^{T}\langle A u(s), z(s)\rangle d s \leq \mathbb{E} \int_{0}^{T}\left\langle a^{\infty}(s), z(s)\right\rangle d s
$$

This holds for an arbitrary $z \in \mathcal{L}^{p}(V)$ and hence, in particular, for $-z$. Thus one obtains that $a^{\infty}=A u$.

Due to Theorem 1, the solution $u$ to (1.1) is unique. Thus the whole sequences of approximations converges rather than just the subsequence denoted by $\ell^{\prime}$.

Finally, in order to show that $u_{\ell}(T) \rightarrow u(T)$ in $L^{2}(\Omega ; H)$, one uses (4.6) and (4.7) with $w=u$ together with $a^{\infty}=A u$ and $b^{\infty}=B u$. Consequently, the weak-lowersemi-continuity of the norm and Lemma 2 lead to

$$
\begin{aligned}
e^{-K T} \mathbb{E}|u(T)|^{2} \leq & \liminf _{\ell \rightarrow \infty} e^{-K T} \mathbb{E}\left|u_{\ell}(T)\right|^{2} \leq \mathbb{E}\left|u_{0}\right|^{2}-K \mathbb{E} \int_{0}^{T} e^{-K s}|u(s)|^{2} d s \\
& +\mathbb{E} \int_{0}^{T} e^{-K s}\left[2\langle A u(s), u(s)\rangle+\|B u(s)\|_{L_{2}(U, H)}^{2}\right] d s .
\end{aligned}
$$

Thus, due to (4.8),

$$
0 \leq \liminf _{\ell \rightarrow \infty} \mathbb{E}\left|u_{\ell}(T)\right|^{2}-\mathbb{E}|u(T)|^{2} \leq 0 .
$$

From Lemmas 2 and 3, one already knows that $u_{\ell}(T) \rightarrow u(T)$ in $L^{2}(\Omega ; H)$. This is a uniformly convex space (as it is a Hilbert space). Thus one concludes that $u_{\ell}(T) \rightarrow$ $u(T)$ in $L^{2}(\Omega ; H)$. For this see, e.g., Brézis [2, Proposition 3.32].

\section{Examples}

In this section we give examples of three equations which fit into our framework. In all three examples the interpolation inequality is a consequence of the GagliardoNirenberg inequality (see, for example, [15, Theorem 1.24]). The first example is the equation:

$$
d u=\left[\nabla a(\nabla u)-|u|^{p-2} u\right] d t+u d W \text { on } \mathscr{D} \times(0, T)
$$


with $u=0$ on the boundary of the domain $\mathscr{D}$ and $u(\cdot, 0)=u_{0}$ given. Here $a: \mathbb{R}^{d} \rightarrow$ $\mathbb{R}^{d}$ can be nonlinear but it is assumed to be continuous, monotone and growing at most linearly. If we take $a_{i}(z)=z_{i}$ then $\nabla a(\nabla u)=\Delta u$ and this equation is the stochastic Ginzburg-Landau equation. An example of a nonlinear function is $a_{i}(z)=\frac{2+\exp \left(-z_{i}\right)}{1+\exp \left(-z_{i}\right)}$. Moreover $\mathscr{D}$ is a bounded Lipschitz domain in $\mathbb{R}^{d}, d=1,2,3$ and $p \in[2,6)$ if $d=1, p \in[2,4)$ if $d=2$ and $p \in[2,10 / 3)$ if $d=3$. In our framework $H=L^{2}(\mathscr{D})$, $V_{1}=H_{0}^{1}(\mathscr{D})$ and $V_{2}=L^{p}(\mathscr{D})$ (using the standard notation for Lebesgue and Sobolev spaces).

The second is the stochastic Swift-Hohenberg equation:

$$
d u=\left[\left(\gamma^{2}-(1+\Delta)^{2}\right) u-|u|^{p-2} u\right] d t+d W \text { on } \mathscr{D} \times(0, T)
$$

with appropriate boundary and initial conditions. The domain $\mathscr{D}$ is assumed to be a bounded Lipschitz domain in $\mathbb{R}^{2}$. With Dirichlet boundary conditions we would take $V_{1}=H_{0}^{2}(\mathscr{D})$ and $V_{2}=L^{p}(\mathscr{D})$ with $p \in[2,6)$.

The third example is the spatially extended stochastic FitzHugh-Nagumo system for signal propagation in nerve cells (originally stated by FitzHugh [3] as a system of ordinary differential equations, see Bonaccorsi and Mastrogiacomo [1] for mathematical analysis of the spatially extended stochastic version):

$$
\begin{aligned}
& d u=\left(\Delta u+u-u^{3}-v\right) d t+d W \\
& d v=c_{1}\left(u-c_{2} v+c_{3}\right) d t
\end{aligned} \quad \text { on } \quad(0,1) \times(0, T)
$$

together with appropriate initial data for $u$ and $v$ as well as homogeneous Neumann boundary conditions for $u$ only. In this situation $V_{1}=H^{1}((0,1)) \times L^{2}((0,1))$ while $V_{2}=L^{4}((0,1)) \times L^{2}((0,1))$.

We now provide estimates on the constant $\mathfrak{c}(m)$ in the particular case when $\mathscr{D}=$ $(0, \pi)^{2} \subset \mathbb{R}^{2}$ and we use a spectral Galerkin method to construct the spaces $V_{m}$. To that end define

$$
\varphi_{n_{1} n_{2}}\left(x_{1}, x_{2}\right):=\frac{2}{\pi} \sin \left(n_{1} x_{1}\right) \sin \left(n_{2} x_{2}\right) .
$$

Let $V_{m}=\operatorname{span}\left\{\varphi_{n_{1} n_{2}}: n_{1}=1, \ldots, m, n_{2}=1, \ldots, m\right\}$. Then

$$
\Pi_{m} f:=\sum_{n_{1}=1, n_{2}=1}^{m}\left\langle f, \varphi_{n_{1} n_{2}}\right\rangle \varphi_{n_{1} n_{2}}
$$

satisfies Assumption 2. Moreover we can calculate

$$
\begin{aligned}
\mathfrak{c}(m) & =\sum_{n_{1}=1, n_{2}=1}^{m}\left(\left\|\varphi_{n_{1} n_{2}}\right\|_{L^{2}(\mathscr{D})}^{2}+\left\|\nabla \varphi_{n_{1} n_{2}}\right\|_{L^{2}\left(\mathscr{D} ; \mathbb{R}^{2}\right)}^{2}+\left\|\varphi_{n_{1} n_{2}}\right\|_{L^{p}(\mathscr{D})}^{2 / p}\right) \\
& =m^{2}\left(1+2+c_{p}\right)
\end{aligned}
$$


where $c_{p}$ depends only on $p$. Hence, in order to apply Theorem 2, we need a sequence $\left(m_{\ell}, n_{\ell}, k_{\ell}\right)$ such that $\frac{m_{\ell}^{2}}{n_{\ell}} \rightarrow 0$ as $\ell \rightarrow \infty$. This means that we need to choose $n_{\ell}=\left\lfloor m_{\ell}^{2+\delta}\right\rfloor$ for some $\delta>0$.

We also note that if $\mathscr{D}=(0, \pi)^{d}$ then an analogous construction of $V_{m}$ would lead to the conclusion that we need $n_{\ell}=\left\lfloor m_{l}^{d+\delta}\right\rfloor$ for some $\delta>0$. Crucially we see that the space-time coupling requirement is no more onerous than in the case of equations with operators growing at most linearly.

Acknowledgments The authors would like to thank the referees for their comments on the paper.

Open Access This article is distributed under the terms of the Creative Commons Attribution 4.0 International License (http://creativecommons.org/licenses/by/4.0/), which permits unrestricted use, distribution, and reproduction in any medium, provided you give appropriate credit to the original author(s) and the source, provide a link to the Creative Commons license, and indicate if changes were made.

\section{References}

1. Bonaccorsi, S., Mastrogiacomo, E.: Analysis of the stochastic FitzHugh-Nagumo system. Infin. Dimens. Anal. Quantum Probab. Relat. Top. 11(03), 427-446 (2008)

2. Brézis, H.: Functional Analysis. Sobolev spaces and partial differential equations. Springer, New York (2010)

3. FitzHugh, R.: Impulses and physiological states in theoretical models of nerve membrane. Biophys. J. 1, 445-466 (1961)

4. Gajewski, H., Gröger, K., Zacharias, K.: Nichtlineare Operatorgleichungen und Operatordifferentialgleichungen. Akademie-Verlag, Berlin (1974)

5. Giles, M.B.: Multilevel Monte Carlo path simulation. Oper. Res. 56, 607-617 (2008)

6. Gyöngy, I., Millet, A.: On discretization schemes for stochastic evolution equations. Potential Anal. 23(2), 99-134 (2005)

7. Heinrich, S.: Monte Carlo complexity of global solution of integral equations. J. Complex. 14(2), 151-175 (1998)

8. Heinrich, S.: Multilevel Monte Carlo methods. In: Large-Scale Scientific Computing. Lecture Notes in Computer Science, vol. 2179, pp. 58-67. (2001)

9. Hutzenthaler, M., Jentzen, A., Kloeden, P.E.: Strong and weak divergence in finite time of Euler's method for stochastic differential equations with non-globally Lipschitz continuous coefficients. Proc. R. Soc. A 467, 1563-1576 (2011)

10. Hutzenthaler, M., Jentzen, A., Kloeden, P.E.: Strong convergence of an explicit numerical method for SDEs with non-globally Lipschitz continuous coefficients. Ann. Appl. Probab. 22, 1611-1641 (2012)

11. Hutzenthaler, M., Jentzen, A., Kloeden, P.E.: Divergence of the multilevel Monte Carlo Euler method for nonlinear stochastic differential equations. Ann. Appl. Probab. 23(5), 1913-1966 (2013)

12. Hutzenthaler, M., Jentzen, A.: Numerical Approximations of stochastic differential equations with non-globally Lipschitz coefficients. Mem. Amer. Math. Soc. 4, 1-112 (2015)

13. Pardoux, E.: Équations aux dérivées partielles stochastiques non linéaires monotones. Étude de solutions fortes de type Ito. Ph.D. thesis, Univ. Paris XI, Orsay (1975)

14. Roberts, G.O., Tweedie, R.L.: Exponential convergence of Langevindistributions and their discrete approximations. Bernoulli 2(4), 341-363 (1996)

15. Roubíček, T.: Nonlinear partial differential equations with applications. Birkhäuser, Basel (2005)

16. Sabanis, S.: A note on tamed Euler approximations. Electron. Commun. Probab. 18(47), 1-10 (2013)

17. Sabanis, S.: Euler approximations with varying coefficients: the case of superlinearly growing diffusion coefficients. Ann. Appl. Probab. arXiv:1308.1796 (2015)

18. Dareiotis, K., Kumar, C., Sabanis, S.: On Tamed Euler Approximations of SDEs Driven by Lévy Noise with Applications to Delay Equations. arXiv:1403.0498 (2015)

19. Tretyakov, M.V., Zhang, Z.: A fundamental mean-square convergence theorem for SDEs with locally Lipschitz coefficients and its applications. SIAM J. Numer. Anal. 51(6), 3135-3162 (2013) 